\title{
Effect of Expiratory Muscle Fatigue on the Respiratory Response during Exercise
}

\author{
Hiromichi Sugiura, PT, MS ${ }^{1,2)^{*}}$, Shunji Sako, PT, MS ${ }^{1)}$, Yoshiharu Oshida, MD, PhD2) \\ 1) Department of Physical Therapy, Nagoya Isen: 4-27-1 Meieki, Nakamura-ku, Nagoya 450-0002, \\ Japan \\ 2) Graduate School of Medicine, Nagoya University, Japan
}

\begin{abstract}
Purpose] The aim of this study was to reveal the effect of expiratory muscle fatigue (EMF) on respiratory response under two different exercise conditions: exercise (EX) with EMF (EMF-EX) and control EX without EMF (CON-EX). [Methods] Nine healthy adult men performed cycle exercise with a ramp load, and a spirometer was used to measure forced vital capacity (FVC), forced expiratory volume in one second, percent of forced expiratory volume, maximal expiratory mouth pressure, and maximal inspiratory mouth pressure $\left(\mathrm{PI}_{\max }\right)$ to evaluate respiratory functions immediately and at 15 and $30 \mathrm{~min}$ after exercise. To assess the respiratory response during exercise, an exhaled gas analyzer was used to measure minute ventilation $\left(V_{E}\right)$, respiratory frequency (f), tidal volume $\left(\mathrm{V}_{\mathrm{T}}\right)$, oxygen uptake, and carbon dioxide output. In addition, the Borg Scale was used to evaluate dyspnea, while electrocardiography was used to measure heart rate. [Results] The results showed that compared with the CON-EX condition, no change in $\mathrm{V}_{\mathrm{E}}$, an increase in $\mathrm{f}$, or a decrease in $\mathrm{V}_{\mathrm{T}}$ was observed under the medium-intensity EMF-EX condition, while high-intensity exercise reduced $V_{E}$ and $f$ without changing $V_{T}$. [Conclusion] These results suggest that during medium-intensity exercise, EMF modulates the respiratory response by inducing shallow and fast breathing to increase ventilation volume.
\end{abstract}

Key words: Expiratory muscle fatigue, Maximal mouth pressure, Respiratory response

(This article was submitted May 21, 2013, and was accepted Jun. 24, 2013)

\section{INTRODUCTION}

At rest, inspiratory muscle activity is dominant with little or no expiratory muscle activity. During exercise, breathing is hyperventilatory and requires expiratory muscle activity as well as an increase in inspiratory muscle activity ${ }^{1-3)}$. Respiratory muscle activity plays an important role in ventilation control and is especially essential for the respiratory response during exercise. Fatigue in respiratory muscles can lead to dyspnea and increased forced respiration. Several studies have shown that expiratory ${ }^{4,5)}$ and inspiratory ${ }^{6-9)}$ muscles are fatigued during high-intensity exercise, and such fatigue may thus affect exercise tolerance. Respiratory muscle training is widely performed not only by athletes but also in rehabilitation for patients with respiratory diseases. However, the nature of the relationship between respiratory muscles and exercise performance is unclear, and expiratory muscles have not been studied separately. It has been reported that expiratory muscles are more prone than inspiratory muscles to fatigue ${ }^{10,11)}$ because the activity of expiratory muscles increases with an increase in exercise

*Corresponding author. Hiromichi Sugiura (e-mail: sugiura. hiromichi@gmail.com)

(C)2013 The Society of Physical Therapy Science

This is an open-access article distributed under the terms of the Creative Commons Attribution Non-Commercial No Derivatives (by-nc-nd) License $<\mathrm{http}$ ://creativecommons.org/ licenses/by-nc-nd/3.0/>. intensity ${ }^{12)}$ and because expiratory muscle fatigue (EMF) enhances neuromuscular activity ${ }^{13)}$, suggesting that EMF affects the respiratory response during exercise. In this study, we investigated the effect of EMF on the respiratory response during incremental ramp cycle exercise.

\section{SUBJECTS AND METHODS}

The subjects were 9 healthy adult men (mean age 21.0 \pm 0.5 years, mean body height $171.2 \pm 2.3 \mathrm{~cm}$, mean body weight $64.8 \pm 5.7 \mathrm{~kg}$, mean body mass index (BMI) $22.1 \pm$ $1.8 \mathrm{~kg} / \mathrm{m}^{2}$ ), who did not smoke and were not athletes. We confirmed that none of the subjects had suffered any respiratory or cardiovascular disease within the past 1 month. This protocol was conducted in accordance with the ethical standards of the Declaration of Helsinki. All subjects were given explanations about the details and risks of the study and were asked to sign a consent form prior to participation in the study.

The following body composition parameters were measured before starting exercise using a body composition meter (BC-118E, Tanita, Tokyo, Japan): body weight, percent body fat, lean body weight, skeletal muscle mass, and BMI.

The following pulmonary function parameters were measured using a spirometer (AS-507, Minato, Osaka, Japan): $\mathrm{FVC}, \mathrm{FEV}_{1}$, and $\mathrm{FEV}_{1.0 \%}$; as measures of respiratory muscle strength, $\mathrm{PE}_{\max }$ and $\mathrm{PI}_{\max }$ were also measured. The subjects were instructed to hold their cheeks with their hands to prevent air leakage and to keep the trunk stable 
during measurement. At the start of measurement, the subjects were instructed to maintain maximal mouth pressure for $1.5 \mathrm{~s}$ at the maximal expiratory level to measure $\mathrm{PI}_{\max }$ and at the maximal inspiratory level to measure $\mathrm{PE}_{\max }$. The measurements were repeated 10 times. The three highest measurement values with an error of $5 \%$ or less were selected and the mean of the three values was calculated ${ }^{7}$.

During resistive breathing, subjects inspired freely through the nose and expired through a mouthpiece (nose occluded by hand) connected to a resistance mechanism consisting of a closed perspex tube containing three holes that were $0.5-1 \mathrm{~mm}$ in diameter. Subjects had to expire through this device and achieve a target pressure corresponding to at least $50 \%$ of their individual $\mathrm{PE}_{\max }$, similar to previous studies ${ }^{14-16)}$.

The target expiratory pressure was displayed to the subjects on a spirometer display and had to be maintained as a square wave. The expiratory duty cycle was 0.8 , and the breathing frequency was set to 12 breaths/min, corresponding to a spontaneous breathing pattern during expiratory resistive breathing ${ }^{17)}$. Subjects used a "mask" developed in our laboratory to prevent leaks at the mouthpiece and puffing up of the cheeks during forced expiration. After the subject performed this expiration activity continuously for 20 min, $\mathrm{PE}_{\max }$ was assessed again. If the reduction of $\mathrm{PE}_{\max }$ was still less than $20 \%$, the subjects were asked to continue breathing against the expiratory resistance, and $\mathrm{PE}_{\max }$ was assessed again after exhaustion or after another $20 \mathrm{~min}$. A $20 \%$ threshold was chosen in order to induce a degree of fatigue similar to that in Mador and Acevedo's study ${ }^{18)}$.

Subjects were instructed to perform cycle exercise using a cycle ergometer (75XL II ME, Combi Wellness, Tokyo, Japan) with the ramp load set at $20 \mathrm{~W} / \mathrm{min}$ and the pedal rotation rate set at 55-60 rpm. The exercise was completed when the pedal rotation rate fell below $55 \mathrm{rpm}$.

As measures of the respiratory response, $\mathrm{V}_{\mathrm{E}}, \mathrm{f}, \mathrm{V}_{\mathrm{T}}, \mathrm{VO}_{2}$, and $\mathrm{VCO}_{2}$ were measured using an exhaled gas analyzer (AE-300S, Minato, Osaka, Japan) on a breath-by-breath basis. HR was also calculated from R-R intervals on an electrocardiogram (ECG-9522, Nihon Kohden, Tokyo, Japan) as a measure of heart rate response. For the assessment of the maximal respiratory and heart rate responses during exercise, peak $\mathrm{VO}_{2}$ was calculated as the mean value during the 30 -s period before the load reached the maximum; other measures were also calculated in the same fashion. At the end of each $1 \mathrm{~min}$, respiratory exertion was assessed, i.e., the experimenter shouted to the subject "Respiratory exertion?" and the subject shouted out a number between 0 and 10.

Two experiments were performed randomly under different exercise conditions, exercise with EMF (EMF-EX) and exercise without EMF (CON-EX; control), with a $>48-\mathrm{h}$ interval between the experiments. Subjects refrained from caffeine prior to exercise and used a cycle ergometer at $30 \mathrm{~W}$ for $10 \mathrm{~min}$ to warm up. Pulmonary function $\left(\mathrm{FVC}, \mathrm{FEV}_{1}\right.$, and $\left.\mathrm{FEV}_{1.0 \%}\right)$ and maximal mouth pressure $\left(\mathrm{PE}_{\max }\right.$ and PI$\max$ ) were measured before each exercise experiment. Under the EMF-EX conditions, $\mathrm{PE}_{\max }$ and $\mathrm{PI}_{\max }$ were measured again after expiratory muscles were fatigued in accordance with the EMF method, followed by cycle exercise. Under the CON-EX conditions, the pre-exercise measurements of respiratory function and maximal mouth pressure were followed by cycle exercise. After finishing the load exercise, subjects were required to continue the cycle exercise without a load for 3 min as a cooldown. Pulmonary function parameters and maximal mouth pressure were then measured immediately, $15 \mathrm{~min}$ and $30 \mathrm{~min}$ after exercise completion.

In the measurement of the respiratory response during cycle exercise, the maximum exercise load with the same exercise duration under the EMF-EX and CON-EX conditions was defined as high-intensity exercise, $\mathrm{W}_{100 \%}$, and half the exercise load was defined as medium-intensity exercise, $\mathrm{W}_{50 \%}$. $\mathrm{V}_{\mathrm{E}}, \mathrm{f}, \mathrm{V}_{\mathrm{T}}, \mathrm{VO}_{2}$, and $\mathrm{VCO}_{2}$ at $\mathrm{W}_{100 \%}$ and $\mathrm{W}_{50 \%}$ were compared between the two conditions. Borg Scale scores were compared for minute by minute changes during the 10-min period in which data from all subjects were available. To examine the effect of exercise on pulmonary function and mouth pressure, pulmonary function parameters and maximal mouth pressure were compared before and after exercise. Friedman's $\chi^{2}$ r-test was used to compare data, and significant differences $(\mathrm{p}<0.05)$ were further analyzed with Dunnett's test and Wilcoxon's t-test. All statistical analyses were performed using statistical analysis software, SPSS for Windows (version 16. 0.2 J).

\section{RESULTS}

Under the EMF-EX and CON-EX conditions, no significant change in $\mathrm{FVC}, \mathrm{FEV}_{1}$, or $\mathrm{FEV}_{1.0 \%}$ was observed immediately or at 15 or 30 min after exercise compared with the before exercise values (Table 1).

EMF-EX $\mathrm{PE}_{\max }$ was significantly reduced after EMF and immediately and $15 \mathrm{~min}$ after cycle exercise compared with the pre-exercise values, whereas EMF-EX PI max $_{\text {showed no }}$ significant differences after EMF or exercise. In addition, CON-EX $\mathrm{PE}_{\max }$ and $\mathrm{PI}_{\max }$ showed no significant differences immediately after exercise compared with the pre-exercise values (Table 2). The mean exercise durations were $573.7 \pm$ 51.9 and $545.1 \pm 105.0 \mathrm{~s}$ under the EMF-EX and CON-EX conditions, respectively, with no significant differences observed between the conditions.

Compared with CON-EX, EMF-EX $\mathrm{V}_{\mathrm{E}}$ was significantly reduced at $\mathrm{W}_{100 \%}$, with no change at rest or $\mathrm{W}_{50 \%}$, whereas EMF-EX $\mathrm{f}$ was increased at $\mathrm{W}_{50 \%}$ but decreased at $\mathrm{W}_{100 \%}$, with no change at rest. Furthermore, EMF-EX $V_{\mathrm{T}}$ was significantly reduced at $\mathrm{W}_{50 \%}$, with no change at rest or $\mathrm{W}_{100 \%}$. There was no significant difference in $\mathrm{VO}_{2}, \mathrm{VCO}_{2}$, or $\mathrm{HR}$ at rest, $\mathrm{W}_{50 \%}$, or $\mathrm{W}_{100 \%}$ between the two conditions (Table 3 ).

Compared with CON-EX, Borg Scale scores obtained under the EMF-EX conditions started to increase immediately before and 1 and 2 min after exercise, but no significant differences were observed (Table 4).

\section{DISCUSSION}

Although EMF caused a reduction in $\mathrm{V}_{\mathrm{T}}$ during mediumintensity exercise, an increase in $f$ brought the amount of ventilation back to a similar level to that in CON-EX. However, due to a reduction in $\mathrm{f}$ during high-intensity exercise, 
Table 1. Changes in pulmonary function after exercise

\begin{tabular}{|c|c|c|c|c|c|c|c|c|}
\hline & \multicolumn{2}{|c|}{ Before exercise } & \multicolumn{6}{|c|}{ After exercise } \\
\hline & \multirow[b]{2}{*}{ EMF } & \multirow[b]{2}{*}{$\mathrm{CON}$} & \multicolumn{2}{|c|}{ Immediately } & \multicolumn{2}{|c|}{$15 \mathrm{~min}$} & \multicolumn{2}{|c|}{$30 \mathrm{~min}$} \\
\hline & & & EMF & $\mathrm{CON}$ & EMF & $\mathrm{CON}$ & EMF & $\mathrm{CON}$ \\
\hline FVC [1] & $4.5(0.5)$ & $4.7(0.4)$ & $4.5(0.5)$ & $4.6(0.5)$ & $4.5(0.5)$ & $4.6(0.4)$ & $4.6(0.4)$ & $4.6(0.5)$ \\
\hline $\mathrm{FEV}_{1.0}[1]$ & $4.0(0.4)$ & $4.2(0.3)$ & $4.1(0.3)$ & $4.2(0.3)$ & $4.0(0.4)$ & $4.1(0.3)$ & $4.1(0.3)$ & $4.1(0.3)$ \\
\hline $\mathrm{FEV}_{1.0} / \mathrm{FVC}[\%]$ & $89.8(8.5)$ & $88.6(5.6)$ & $90.8(6.6)$ & $91.3(6.6)$ & $89.5(8.0)$ & $80.6(7.0)$ & $89.2(7.7)$ & $89.5(7.0)$ \\
\hline
\end{tabular}

All data are expressed as means (SD).

Table 2. Changes in maximal mouth pressures after exercise

\begin{tabular}{lllllll}
\hline & & \multicolumn{3}{c}{ After exercise } \\
\cline { 3 - 7 } & & Before exercise & After EMF & Immediately & \multicolumn{1}{c}{15 min } & 30 min \\
\hline \multirow{2}{*}{ PEmax $\left[\mathrm{cmH}_{2} \mathrm{O}\right]$} & EMF-EX & $201.7(21.2)$ & $177.6(14.3)^{* *}$ & $187.1(16.4)^{* *}$ & $186.0(19.4)^{*}$ & $194.1(19.0)$ \\
& CON-EX & $198.1(24.4)$ & & $179.4(20.3)$ & $180.2(23.1)$ & $188.3(29.8)$ \\
\multirow{2}{*}{ PImax $\left[\mathrm{cmH}_{2} \mathrm{O}\right]$} & EMF-EX & $107.5(13.0)$ & $102.4(22.3)$ & $107.5(28.9)^{* *}$ & $108.4(31.7)^{*}$ & $110.5(31.2)$ \\
& CON-EX & $102.6(30.0)$ & & $96.4(28.1)$ & $100.7(33.2)$ & $105.5(34.3)$ \\
\hline
\end{tabular}

All data are expressed as means (SD). ${ }^{*} \mathrm{p}<0.05,{ }^{* *} \mathrm{p}<0.01$

Table 3. Changes in cardiorespiratory response during exercise

\begin{tabular}{|c|c|c|c|c|c|c|}
\hline & \multicolumn{2}{|c|}{ Rest } & \multicolumn{2}{|c|}{$\mathrm{W}_{50 \%}$} & \multicolumn{2}{|c|}{$\mathrm{W}_{100 \%}$} \\
\hline & EMF-EX & CON-EX & EMF-EX & CON-EX & EMF-EX & CON-EX \\
\hline $\mathrm{V}_{\mathrm{E}}[1 / \mathrm{min}]$ & $12.0(3.7)$ & $10.7(3.8)$ & $36.3(7.0)$ & $36.1(3.6)$ & $\begin{array}{c}79.2(19.7) \\
\llcorner\end{array}$ & $92.3(20.2)$ \\
\hline $\mathrm{f}[$ beats/ min] & $17.0(3.0)$ & $15.2(5.0)$ & $\begin{array}{c}25.7(7.2) \\
\llcorner\end{array}$ & $21.6(5.1)$ & $\begin{array}{c}38.9(8.3) \\
\stackrel{L}{L}\end{array}$ & \begin{tabular}{l}
$44.7(11.2)$ \\
\hdashline
\end{tabular} \\
\hline $\mathrm{V}_{\mathrm{T}}[\mathrm{ml} / \mathrm{min}]$ & $695.1(135.6)$ & $755.2(257.5)$ & $1398.0(261.0)$ & $1630.1(535.7)$ & $2050.3(417.1)$ & $2025.2(405.1)$ \\
\hline $\mathrm{VO}_{2}[\mathrm{ml} / \mathrm{min}]$ & $379.8(102.1)$ & $373.4(101.0)$ & $1371.0(161.3)$ & $1468.0(237.4)$ & $2510.1(302.0)$ & $2615.8(370.1)$ \\
\hline $\mathrm{VCO}_{2}[\mathrm{ml} / \mathrm{min}]$ & $411.3(187.6)$ & 356.4 (89.7) & $1360.9(147.9)$ & $1429.6(157.8)$ & 2999.1 (331.3) & $3228.2(415.7)$ \\
\hline HR [beats/ $\mathrm{min}$ ] & $84.4(12.6)$ & $89.1(14.2)$ & $129.6(7.2)$ & $135.0(13.3)$ & $180.1(11.5)$ & $187.7(7.9)$ \\
\hline
\end{tabular}

All data are expressed as means $(\mathrm{SD})$. $\mathrm{V}_{\mathrm{E}}$, minute ventilation; $\mathrm{f}$, respiratory frequency; $\mathrm{V}_{\mathrm{T}}$, tidal volume; $\mathrm{VO}_{2}$, oxygen uptake; $\mathrm{VCO}_{2}$, carbon dioxide output; $\mathrm{HR}$, heart rate; $* \mathrm{p}<0.05$

Table 4. Changes in respiratory exertion during exercise

\begin{tabular}{ccc}
\hline Time $[\mathrm{min}]$ & EMF-EX & CON-EX \\
\hline 1 & $1.8(1.2)$ & $0.7(0.7)^{*}$ \\
2 & $2.1(0.9)$ & $1.0(1.0)^{*}$ \\
3 & $2.4(0.7)$ & $1.7(1.3)$ \\
4 & $2.8(0.4)$ & $2.4(1.2)$ \\
5 & $3.4(0.5)$ & $3.2(1.3)$ \\
6 & $4.0(0.5)$ & $3.5(1.4)$ \\
7 & $4.6(0.5)$ & $4.4(1.8)$ \\
8 & $5.6(0.9)$ & $4.9(1.8)$ \\
9 & $6.3(1.0)$ & $5.9(2.1)$ \\
10 & $6.8(1.2)$ & $6.0(1.6)$ \\
\hline
\end{tabular}

All data are expressed as means (SD). ${ }^{*} \mathrm{p}<0.05$

the respiratory response failed to increase the ventilation volume to meet the exercise load. These results suggest that although EMF modulates the respiratory pattern to increase ventilation volume during medium-intensity exercise, EMF during high-intensity exercise fails to increase $\mathrm{f}$, resulting in a reduction in ventilation volume.

In the present study, EMF was induced using mouth pressure. To confirm fatigue of the expiratory muscles, the maximal mouth pressure was measured after EMF and after cycle exercise to ensure that the pressure after cycle exercise was less than that before exercise. Because the maximal mouth pressure is affected by residual volume ${ }^{15}$, 17), we also measured $\mathrm{FVC}$ together with $\mathrm{PE}_{\max }$ and $\mathrm{PI}_{\max }$. In this study, post exercise CON-EX FVC was reduced by as much as EMF-EX FVC, suggesting that the reduction was caused by cycle exercise. In addition, vital capacity was similar under both conditions, but EMF-EX $\mathrm{PE}_{\max }$ immediately after EMF and cycle exercise was reduced significantly compared with CON-EX PE $\mathrm{max}_{\text {max }}$ These results indicate that under the EMF-EX conditions, expiratory muscles remained fatigued throughout cycle exercise due to EMF.

In general, $\mathrm{V}_{\mathrm{T}}$ and $\mathrm{f}$ are increased almost simultaneous- 
ly to increase the amount of ventilation during moderate or low-intensity exercise. If $\mathrm{V}_{\mathrm{E}}$ is increases further due to more intense exercise, then $\mathrm{V}_{\mathrm{T}}$ reaches its upper limit, and f increases dramatically ${ }^{19)}$. In the present study, however, an increase in $\mathrm{f}$ and a reduction in $\mathrm{V}_{\mathrm{T}}$ were observed during medium-intensity exercise, indicating that breathing was changed to a rapid shallow pattern under the EMF-EX conditions. Previous studies on the respiratory response due to EMF have reported an increase in $\mathrm{f}$ due to fatigue of the diaphragm ${ }^{20)}$ and rapid shallow breathing during exercise after EMF ${ }^{21,22)}$, which are in accord with the present results. During exercise, expiratory muscles function not only to increase $f$ but also to increase inspiratory volume by reducing residual volume in the lung and expanding the diaphragm $\left.{ }^{2}, 13,23\right)$. Because the involvement of expiratory muscles in the ventilation volume increases during exercise, EMF may reduce the amount of ventilation during exercise.

In this study, instead of increasing $\mathrm{V}_{\mathrm{T}}$, $\mathrm{f}$ was increased to bring the ventilation volume to a level similar to that in CON-EX, suggesting that EMF modulated the respiratory control mechanism during medium-intensity EMFEX. Dempsey et al. reported the presence of a regulatory mechanism that optimizes the work efficiency of respiratory movement ${ }^{19)}$, while Poon et al. proposed a theory of optimal respiratory control in which $\mathrm{V}_{\mathrm{T}}$ and $\mathrm{f}$ are regulated to minimize the work load of the main and accessory respiratory muscles ${ }^{24}$. It is therefore likely that EMF affected the regulatory mechanism of the respiratory response during exercise and induced a rapid shallow breathing pattern to reduce the burden of expiratory muscles and thus increase ventilation volume. In addition to respiratory muscle fatigue, a reduction in $\mathrm{PCO}_{2}$ due to hyperventilation and shortness of breath may also affect $\mathrm{V}_{\mathrm{T}}{ }^{25}$. In this study, however, these factors were unlikely to have been involved in the respiratory response during medium- and high-intensity EMF-EX because there were no significant changes in $\mathrm{VCO}_{2}$, no reductions in $\mathrm{PaCO}_{2}$ due to hyperventilation, and no significant differences in Borg Scale scores, except during the early postexercise phase. These results suggest that the reduction in $V_{T}$ was caused by EMF. With regard to $V_{E}$ during exercise, it was possible to increase ventilation volume in EMF-EX to a level similar to that in CON-EX during medium-intensity, but not high-intensity, exercise. This indicates that the effect of EMF on the respiratory response during medium-intensity exercise can be counteracted by a change in the respiratory pattern to increase ventilation volume, which appears to be difficult during high-intensity exercise.

This study clearly showed that EMF modulates the respiratory response during exercise. Conventional respiratory muscle training in patients with chronic respiratory disease has problems that need to be improved related to such things as shortness of breath ${ }^{26)}$, the ventilation response $\mathrm{e}^{27)}$, and exercise tolerance ${ }^{28}$. Because EMF affects the respiratory response during exercise, we believe that strengthening of the expiratory muscles will enhance the respiratory response during exercise as well as ventilation efficiency in the elderly and in patients with respiratory disease.

Due to EMF, ventilation volume was increased via rapid shallow breathing during exercise. However, during highintensity exercise, the amount of ventilation did not increase to a point sufficient to meet the exercise load because of a failure to increase $\mathrm{f}$. These results suggest that despite the mechanism to regulate breathing patterns through which EMF modulates ventilation volume as a respiratory response during exercise, ventilation volume was reduced during high-intensity exercise.

\section{REFERENCES}

1) Abraham KA, Feingold H, Fuller DD, et al.: Respiratory- related activation of human abdominal muscles during exercise. J Physiol, 2002, 541: 653-663. [Medline] [CrossRef]

2) Henke KG, Sharratt M, Pegelow D, et al.: Regulation of end- expiratory lung volume during exercise. J Appl Physiol, 1988, 64: 135-146. [Medline]

3) Strohl KP, Mead J, Banzett RB, et al.: Regional differences in abdominal muscle activity during various maneuvers in humans. J Appl Physiol, 1981, 51: 1471-1476. [Medline]

4) Laude EA, Duffy NC, Baveystock C, et al.: The effect of helium and oxygen on exercise performance in chronic obstructive pulmonary disease: a randomized crossover trial. Am J Respir Crit Care Med, 2006, 173: 865870. [Medline] [CrossRef]

5) Richardson RS, Sheldon J, Poole DC, et al.: Evidence of skeletal muscle metabolic reserve during whole body exercise in patients with chronic obstructive pulmonary disease. Am J Respir Crit Care Med, 1999, 159: 881-885. [Medline] [CrossRef]

6) Mancini D, Donchez L, Levine S: Acute unloading of the work of breathing extends exercise duration in patients with heart failure. J Am Coll Cardiol, 1997, 29: 590-596. [Medline] [CrossRef]

7) Verges S, Sager Y, Erni C, et al.: Expiratory muscle fatigue impairs exercise performance. Eur J Appl Physiol, 2007, 101: 225-232. [Medline] [CrossRef]

8) Gandevia SC: Spinal and supraspinal factors in human muscle fatigue. Physiol Rev, 2001, 81: 1725-1789. [Medline]

9) Kayser B: Exercise starts and ends in the brain. Eur J Appl Physiol, 2003, 90: 411-419. [Medline] [CrossRef]

10) Verges S, Schulz C, Perret C, et al.: Impaired abdominal muscle contractility after high-intensity exhaustive exercise assessed by magnetic stimulation. Muscle Nerve, 2006, 34: 423-430. [Medline] [CrossRef]

11) St Croix CM, Morgan BJ, Wetter TJ, et al.: Fatiguing inspiratory muscle work causes reflex sympathetic activation in humans. J Physiol, 2000, 529: 493-504. [Medline] [CrossRef]

12) Uribe JM, Stump CS, Tipton CM, et al.: Influence of exercise training on the oxidative capacity of rat abdominal muscles. Respir Physiol, 1992, 88: 171-180. [Medline] [CrossRef]

13) Martin JG, De Troyer A: The behaviour of the abdominal muscles during inspiratory mechanical loading. Respir Physiol, 1982, 50: 63-73. [Medline] [CrossRef]

14) Derchak PA, Sheel AW, Morgan BJ, et al.: Effects of expiratory muscle work on muscle sympathetic nerve activity. J Appl Physiol, 2002, 92: 1539-1552. [Medline]

15) Haverkamp HC, Metelits M, Hartnett J, et al.: Pulmonary function subsequent to expiratory muscle fatigue in healthy humans. Int J Sports Med, 2001, 22: 498-503. [Medline] [CrossRef]

16) Suzuki S, Suzuki J, Ishii T, et al.: Relationship of respiratory effort sensation to expiratory muscle fatigue during expiratory threshold loading. Am Rev Respir Dis, 1992, 145: 461-466.

17) Suzuki S, Suzuki J, Okubo T: Expiratory muscle fatigue in normal subjects. J Appl Physiol, 1991, 70: 2632-2639. [Medline]

18) Mador MJ, Acevedo FA: Effect of respiratory muscle fatigue on breathing pattern during incremental exercise. Am Rev Respir Dis, 1991, 143: 462-468. [Medline] [CrossRef]

19) Dempsey JA, Adams L, Ainsworth DM, et al.: Airway, lung, and respiratory muscle function during exercise. In: Handbook of Physiology, Sec 12, Exercise: Regulation and integration of multiple systems, Rowell LB and Shepherd JT (eds.), New York: Am Physiol Soc, 1996, pp 448-514.

20) Road J, Vahi R, del Rio P, et al.: In vivo contractile properties of fatigued diaphragm. J Appl Physiol, 1987, 63: 471-478. [Medline]

21) Yan S, Sliwinski P, Gauthier AP, et al.: Effect of global inspiratory muscle fatigue on ventilatory and respiratory muscle responses to $\mathrm{CO} 2$. J Appl Physiol, 1993, 75: 1371-1377. [Medline]

22) Verges $S$, Notter $D$, Spengler CM: Influence of diaphragm and rib cage 
muscle fatigue on breathing during endurance exercise. Respir Physiol Neurobiol, 2006, 154: 431-442. [Medline] [CrossRef]

23) Gallagher CG, Hof VI, Younes M: Effect of inspiratory muscle fatigue on breathing pattern. J Appl Physiol, 1985, 59: 1152-1158. [Medline]

24) Poon CS: Ventilatory control in hypercapnia and exercise: optimization hypothesis. J Appl Physiol, 1987, 62: 2447-2459. [Medline]

25) Syabbalo NC, Krishnan B, Zintel T, et al.: Differential ventilator contro during constant work rate and incremental exercise. Respir Physiol, 1994 97: 175-187. [Medline] [CrossRef]
26) Mahler DA, Weinberg DH, Wells CK, et al.: The measurement of dyspnea. Contents, interobserver agreement, and physiologic correlates of two new clinical indexes. Chest, 1984, 85: 751-758. [Medline] [CrossRef]

27) Stoller JK, Ferranti R, Feinstein AR, et al.: Further specification and evaluation of a new clinical index for dyspnea. Am Rev Respir Dis, 1986, 134: 1129-1134. [Medline]

28) Macklem PT, Roussos CS: Respiratory muscle fatigue: a cause of respiratory failure? Clin Sci Mol Med, 1977, 53: 419-422. [Medline] 